\title{
Research on Crowd Behavior Based on the Velocity Field Yuan Fang ${ }^{1, a}$, Ma Ke ${ }^{2}$, Liang Jia-hong ${ }^{3}$ and Chao Yong-jing ${ }^{4}$ \\ 1,3,4 College of Information Systems and Management \\ National University of Defense Technology \\ ${ }^{2}$ Aviation University of Air Force \\ a15575138160@163.com
}

\begin{abstract}
Keywords: crowd behavior, velocity field, Gaussian filter, Horn-Schunck algorithm, interpolation
\end{abstract} function.

\begin{abstract}
Data-driven modeling method has become an ideal research method in crowd behavior simulation fields. The basic idea of data-driven modeling method is that velocity field generated by the crowd behavior characteristic information. Because of the video data acquisition, the velocity field has its limitation, namely virtual crowd simulation scenario should be consistent with the crowd scene of video data. So we present an optimization strategy: first, it eliminates velocity of scene from the generated velocity field; And second, it constructs velocity field by interpolation again. Experiments show that the presented optimization strategy can design the velocity field without scene information, and it is helpful to improve the universality of the velocity field.
\end{abstract}

\section{Introduction}

At present, research on crowd behavior has become a hot topic in the public emergency evacuation, military action, and the social security, etc. In the large-scale crowd activity scene, data-driven modeling method has become an effective research method. It was first put forward in 2007 by Musse SR[1]. Using computer vision methods, he puts forward a method to construct crowd motion path (that is, complete velocity field). The method has two steps: first, it extracted crowd flow movement information from the video data; and second, velocity field generated by characteristic information and interpolation . The method has many advantages[2]: no need to be the initial parameter settings, covering a large number of individual motion information, high efficiency, strong image fidelity. While, there are still some limitations: virtual crowd simulation scenario should be consistent with the crowd scene of video data.

Many scholars have been began to pay attention to the research and application of data-driven model, and all kinds of optimization methods have been put forward. N.Courty[3] presents a crowd velocity field model by collecting from real people video and composing of a series of time series. Andrade[4] puts forward a crowd behavior analysis method using Hidden Markov model in emergency scenarios, and he adopts the visual information features instead of path strategies to extract the crowd features in the video stream. By gathering crowd movement of the real environment, Lerner[5] puts the crowd behavior video separation in time order to extract the sample collection crowd behavior. In this paper, combined with the results of many scholars, we present an optimization method on the limitations that virtual crowd simulation scenario should be consistent with the crowd scene of video data, namely eliminating the scene information from the velocity field and using mathematical interpolation method to design the velocity field.

The rest of paper is organized as follows. Section 2 generates the velocity field. First, we acquire and process the crowd video, and then, we use the optical flow method and Horn-Schunck algorithm to extract the velocity field. Section 3 mainly designs the behavior pattern on the constructed velocity field. First, we optimize the limitations of velocity field, and then, we design the behavior pattern using mathematical interpolation method. Section 4 reports experiments with our planner on Changsha railway station square scenario. Section 5 summarizes the main results and points out direction for future research. 


\section{Behavior patterns extraction on the velocity field}

Video acquisition and processing. We acquire the crowd video in the Changsha railway station square. And aim to the interested areas, we process the data by adjusting the pixel and clipping, etc. Finally, we generate the crowd velocity field.

During the experiment, we found that directly generated velocity field is not very ideal. Mainly embodied in the following aspects: some areas without individual motion still has tiny velocity, result that movement characteristics of pedestrians can not very well by research. Through access to relevant data, we think the above situation is caused by the existence of the image noise. That is to say, when the image data has being processed(recording, adjusting, clipping and encoding etc), it has been interfered in internal or external information. The image noise interfere in the image data, and it has a certain influence on the generation of velocity field. So we again process the data to eliminate noise. The methods of eliminating noise and smoothing have median filter, average filter and Gaussian filter etc. Because Gaussian filter has the advantage of enhancing image effect, eliminating noise and smoothing image, we take Gaussian filter as the experiment method.

Gaussian filter is a process for the calculation of the weighted average, from the view of mathematical properties, it is made convolution of image with normal distribution. One-dimension Gaussian distribution formula is as follows:

$$
G(x)=\frac{1}{\sqrt{2 \pi} \sigma} e^{-\frac{x^{2}}{2 \sigma^{2}}}
$$

Two-dimension Gaussian distribution formula is as follows:

$$
G(x)=\frac{1}{\sqrt{2 \pi} \sigma^{2}} e^{-\frac{x^{2}+y^{2}}{2 \sigma^{2}}}
$$

While $\sigma$ is standard deviation for normal distribution.

Fig. 1 is two-dimension Gaussian distribution diagram.

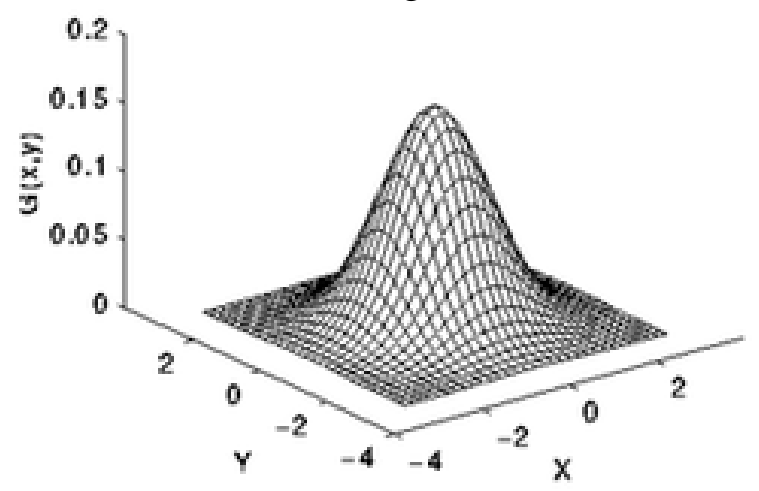

Fig.1 two-dimension Gaussian distribution

Velocity field generation algorithm. We need to estimate the crowd velocity from the video data. And the methods have block matching algorithm, optical flow and motion history image method etc. Between optical flow and motion history image method are often used methods in crowd behavior simulation field. In this paper, we take optical flow to estimate the crowd velocity. Detailed algorithm is as follows.

Optical flow constraint equation. Assume the grey value of point $\mathbf{m}=(x, y)^{T}$ on the image is $I(x, y, t)$ at time $t$, the grey value of it is $I(x+d x, y+d y, t+d t)$ at time $t+d t$. When $d t \rightarrow 0$, we can think the grey value of point on the image has no change, namely:

$$
I(x+d x, y+d y, t+d t)=I(x, y, t)
$$


If the image grey value has no change as $x, y, t$, We can be the left side of expansion Eq.1 used by Taylor formula:

$$
I(x+d x, y+d y, t+d t)=I(x, y, t)+\frac{\partial I}{\partial x} d x+\frac{\partial I}{\partial y} d y+\frac{\partial I}{\partial t} d t+\varepsilon
$$

We give $\varepsilon$ representation for second-order infinitesimal. Because of $d t \rightarrow 0$ and ignoring the value of $\varepsilon$, we can find:

$$
\frac{\partial I}{\partial x} d x+\frac{\partial I}{\partial y} d y+\frac{\partial I}{\partial t} d t=0
$$

While we give $u=\frac{d x}{d t}, v=\frac{d y}{d t}$ representation for optical flow on $\mathrm{x}$ axis and $\mathrm{y}$ axis. And we give $I_{x}=\frac{\partial I}{\partial x}, I_{y}=\frac{\partial I}{\partial y}, I_{t}=\frac{\partial I}{\partial t}$ representation for the image grey value of $x, y, t$ partial derivatives. We can rewrite Eq.5:

$$
I_{x} u+I_{y} v+I_{t}=0
$$

Eq.6 is the basic equation of optical flow field. The vector form of it is as follows:

$$
\nabla \mathbf{I} \cdot \mathbf{v}_{m}+I_{t}=0
$$

While we give $\nabla \mathbf{I}=\left(I_{x}, I_{y}\right)$ representation for gradient of point $\mathrm{m}$ on the image, and we give $\mathbf{v}_{m}=(u, v)$ representation for optical flow of point $m$. Eq.7 is optical flow constraint equation.

Horn-Schunck algorithm. Optical flow constraint equation includes two variable $u$ and $v$, while equation 6 can not get solution, so we need to increase other constraints. And Horn-Schunck algorithm[6] is introduced into the image smoothing constraint equations, namely each pixel of the optical flow smoothly change on the whole image, associated with each other. We take Horn-Schunck algorithm as a method of global constraints.

Assume smoothness constraint is:

$$
E_{s}=\iint\left(u_{x}^{2}+u_{y}^{2}+v_{x}^{2}+v_{y}^{2}\right) d x d y
$$

And

$$
E_{c}=\iint\left(I_{x} u+I_{y} v+I_{t}\right)^{2} d x d y
$$

From the basic formula, we require minimization obviously. So Eq.8 and Eq.9 should satisfy Eq.10:

$$
\min \iint\left[\lambda\left(u_{x}^{2}+u_{y}^{2}+v_{x}^{2}+v_{y}^{2}\right)+\left(I_{x} u+I_{y} v+I_{t}\right)^{2}\right] d x d y
$$

In order to satisfy the needs of minimization, we take derivative of $u$ and $v$ on Eq.10 ,and the reciprocal is zero. Given:

$$
\begin{aligned}
& I_{x}^{2} u+I_{x} I_{y} v=-\lambda^{2} \nabla u-I_{x} I_{t} \\
& I_{y}^{2} v+I_{x} I_{y} u=-\lambda^{2} \nabla v-I_{y} I_{t}
\end{aligned}
$$

Eq.11 and Eq.12 is Euler equation. Assume $\bar{u}$ and $\bar{v}$ give $u$ and $v$ representation for mid-value of their field. Assume $\nabla u=u-\bar{u}$ and $\nabla v=v-\bar{v}$, the Eq.11 and Eq.12 can rewrite as follows: 


$$
\begin{aligned}
& I_{t}=\frac{1}{4}\left(I_{i, j, k+1}+I_{i+1, j, k+1}+I_{i, j+1, k+1}+I_{i+1, j+1, k+1}\right)-\frac{1}{4}\left(I_{i, j, k}+I_{i+1, j, k}+I_{i, j+1, k}+I_{i+1, j+1, k}\right) \\
& \left(I_{x}^{2}+\lambda^{2}\right) u+I_{x} I_{y} v=\lambda^{2} \bar{u}-I_{x} I_{t} \\
& \left(I_{y}^{2}+\lambda^{2}\right) v+I_{x} I_{y} u=\lambda^{2} \bar{v}-I_{y} I_{t}
\end{aligned}
$$

We can find:

$$
\begin{aligned}
& u=\bar{u}-\frac{I_{x}\left(I_{x} \bar{u}+I_{y} \bar{v}+I_{t}\right)}{\lambda^{2}+I_{x}^{2}+I_{y}^{2}} \\
& v=\bar{v}-\frac{I_{y}\left(I_{x} \bar{u}+I_{y} \bar{v}+I_{t}\right)}{\lambda^{2}+I_{x}^{2}+I_{y}^{2}}
\end{aligned}
$$

Eq.16 and Eq.17 are the basic of solving $u$ and $v$ used iterative method. In usually applications, we often take relaxation equation to solve:

$$
\begin{aligned}
u^{(k+1)} & =\bar{u}^{(k)}-I_{x} \frac{I_{x} \bar{u}^{(k)}+I_{y} \bar{v}^{(k)}+I_{t}}{\lambda^{2}+I_{x}^{2}+I_{y}^{2}} \\
v^{(k+1)} & =\bar{v}^{(k)}-I_{y} \frac{I_{x} \bar{u}^{(k)}+I_{y} \bar{v}^{(k)}+I_{t}}{\lambda^{2}+I_{x}^{2}+I_{y}^{2}}
\end{aligned}
$$

While $\mathrm{k}$ is cycle index, and $u^{(0)}$ and $v^{(0)}$ are initial value. They can be zero. $\bar{u}$ and $\bar{v}$ is the mid-value of local image. $\lambda$ is weight coefficient, and it determined based on the derivation of the numerical value.

In the process of usually solving calculation, we need to estimate the differential time and space of image brightness. It can be estimated on the $2 \times 2 \times 2$ field of one point on the image. Assume subscript $i, j, k$ correspond to $x, y, t$ separately, so their one-order partial derivative are:

$$
\begin{aligned}
& I_{x}=\frac{1}{4}\left(I_{i+1, j, k}+I_{i+1, j+1, k}+I_{i+1, j, k+1}+I_{i+1, j+1, k+1}\right)-\frac{1}{4}\left(I_{i, j, k}+I_{i, j+1, k}+I_{i, j, k+1}+I_{i, j+1, k+1}\right) \\
& I_{y}=\frac{1}{4}\left(I_{i, j+1, k}+I_{i+1, j+1, k}+I_{i, j+1, k+1}+I_{i+1, j+1, k+1}\right)-\frac{1}{4}\left(I_{i, j, k}+I_{i+1, j, k}+I_{i, j, k+1}+I_{i+1, j, k+1}\right) \\
& I_{t}=\frac{1}{4}\left(I_{i, j, k+1}+I_{i+1, j, k+1}+I_{i, j+1, k+1}+I_{i+1, j+1, k+1}\right)-\frac{1}{4}\left(I_{i, j, k}+I_{i+1, j, k}+I_{i, j+1, k}+I_{i+1, j+1, k}\right)
\end{aligned}
$$

Namely partial derivative of $x, y, t$ on grey level $I$ represent by one-order differential.

\section{Behavior patterns design on the velocity field}

The velocity field is extracted from video data, and it can study the crowd behavior on Changsha railway station square scenario. While, thinking about universality of the velocity field, we present the optimization design: first, it eliminates velocity of scene from the generated velocity field; And second, it constructs velocity field through interpolation again.

First, we set a threshold velocity $V_{\min }$. When one point velocity is less than $V_{\min }$, we think the point velocity is noise. So ,we can eliminates velocity of scene from the generated velocity field.

And then, we take the crowd movement velocity as sample points, and design velocity field through interpolation. In recent years, a lot of research did on math about function interpolation methods. Studies have shown that Radial Basis Function(RBF) is the most satisfying method in all kinds of interpolation method[7]. So we use the Radial Basis Function interpolation to process sample points, and then the velocity field is obtained. 
Assume we have n sparse velocity, namely the sample points in velocity field, donated $\left\{\left(p_{i}, v_{i}\right)\right\}_{i=1}^{n}$. While $p_{i}=\left(x_{i}, y_{i}\right)$ is an arbitrary point on the plane, $v_{i}=\left(v_{x i}, v_{y i}\right)$ is a velocity vector and correspond to $p_{i}$. We do it for obtaining the arbitrary point velocity vector on area according these sample points, namely data interpolation problem.

We need to find a interpolation function $\left(f^{x}, f^{y}\right):\left(R^{2} \rightarrow R, R^{2} \rightarrow R\right)$, namely given $\operatorname{RBF} \varphi(\theta):(R \rightarrow R)$, finding continuous functions $f^{x}, f^{y}$ :

$$
\begin{aligned}
& f^{x}(x)=\sum_{i=1}^{n} h_{i}^{x} \varphi\left(\left\|x-x_{i}\right\|_{2}\right) \\
& f^{y}(y)=\sum_{i=1}^{n} h_{i}^{y} \varphi\left(\left\|y-y_{i}\right\|_{2}\right)
\end{aligned}
$$

We can find:

$$
\left\{\begin{array}{l}
f^{x}\left(p_{i}\right)=x_{i} \\
f^{y}\left(p_{i}\right)=y_{i}
\end{array} \quad i=1, \cdots, n\right.
$$

RBF can solve $h_{i}^{x}, h_{i}^{y}$.

According to equation 25, we can get continuous functions:

$$
\begin{aligned}
& M_{x} h_{x}=g_{x} \\
& M_{y} h_{y}=g_{y}
\end{aligned}
$$

While $M_{x}$ is n-order interpolation matrix, its elements $m_{i j}^{x}=\varphi\left(\left\|x_{i}-x_{j}\right\|_{2}\right) 1 \leq i, j \leq n$, vector $h_{x}=\left(h_{1}, \cdots, h_{n}\right)^{T}, g_{x}=\left(g_{1}, \cdots, g_{n}\right)^{T}$, and \|\|$_{2}$ is Euclidean distance.

While $M_{x}, M_{y}$ are non-singular matrixes, we can solve the unique solution. But if the distance of sample points is smaller, $M_{x}, M_{y}$ may be ill-conditioned matrixes. We can take anisotropic RBF called by G. Casciola to solve it. The basic idea of anisotropic RBF is that $\left\|M^{\prime}\left(x-x_{i}\right)\right\|_{2}$ is the distance function of each sample points. While $M^{\prime}$ is two-order non-singular square matrix.

\section{Experimental analysis and results}

Video acquisition and processing experimental. We acquire the crowd video in the Changsha railway station square used four rotor aircraft called Phantom3 advanced. Because the required data of optical flow mainly is two images separated by a number of frames. We take the first image(Fig.2, in the red box for the focus area) as example to illustrate process. 


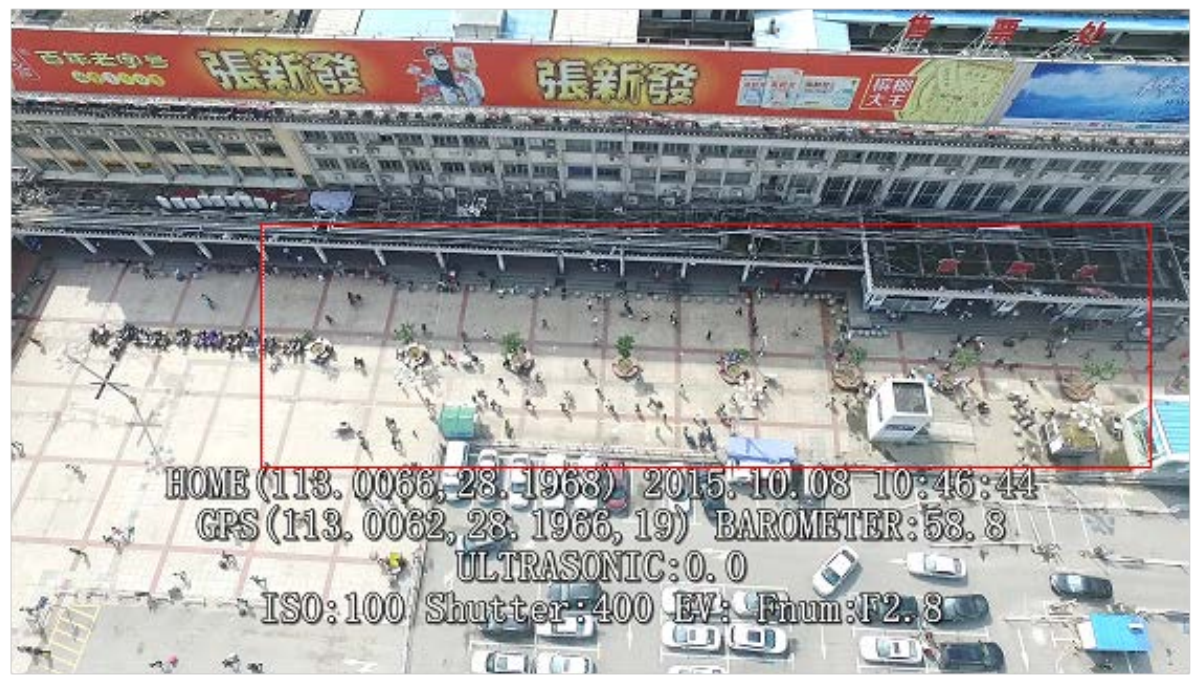

Fig. 2 crowd image

For Fig.2, the image pixel is $1920 * 1080$ and we just study a part of area, so it is meaningless to generate velocity field directly. We process the image before generating velocity field. First, we take image clip from $(457,382)$ to $(1822,735)$; second we adjust its pixel to $430 * 111$. Fig.3 is the processed image.

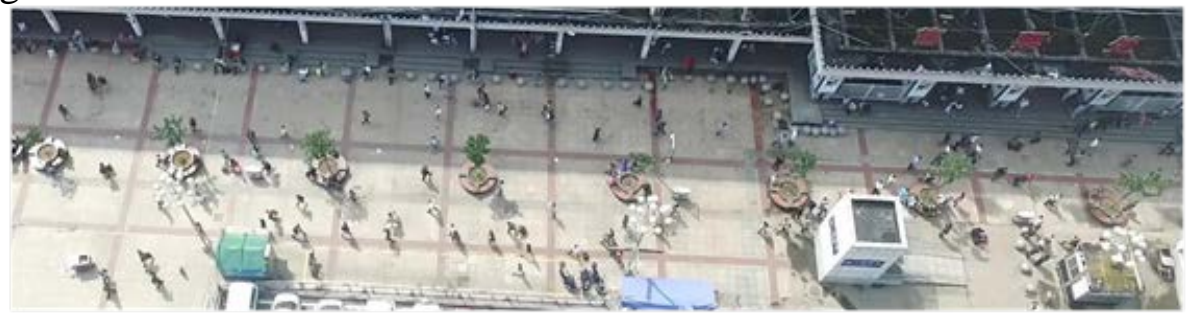

Fig.3 processed image

And then, we eliminate image noise used Gaussian filter. Fig.4 is the finally image.

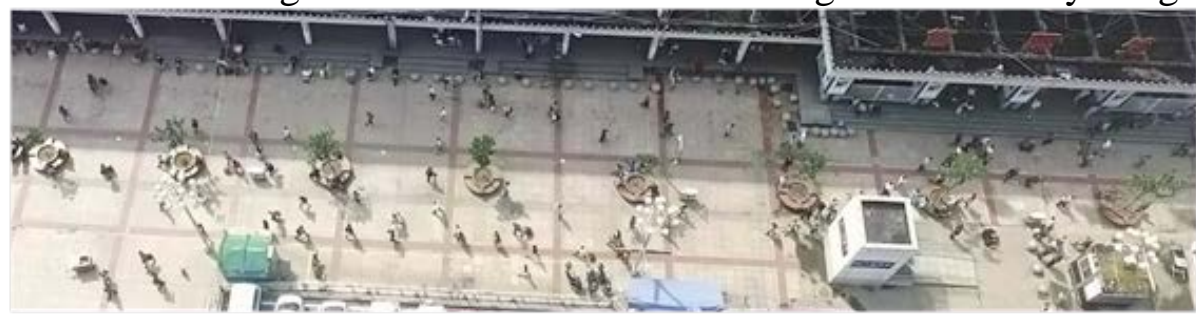

Fig.4 finally image

Form Fig.4, we can see the brightness of the image with the improvement of overall, and the whole image is more soft smooth than before.

Velocity field generation experimental. Experimental do by MATLAB on computer (Intel(R) Core(TM)2 Duo CPU T6600@2.20GHz, 4GB ).

We use Gaussian filter before and after the image constructing velocity field. Fig.5 is velocity field that we use Gaussian filter before the image. Fig.6 is velocity field that we use Gaussian filter after the image.

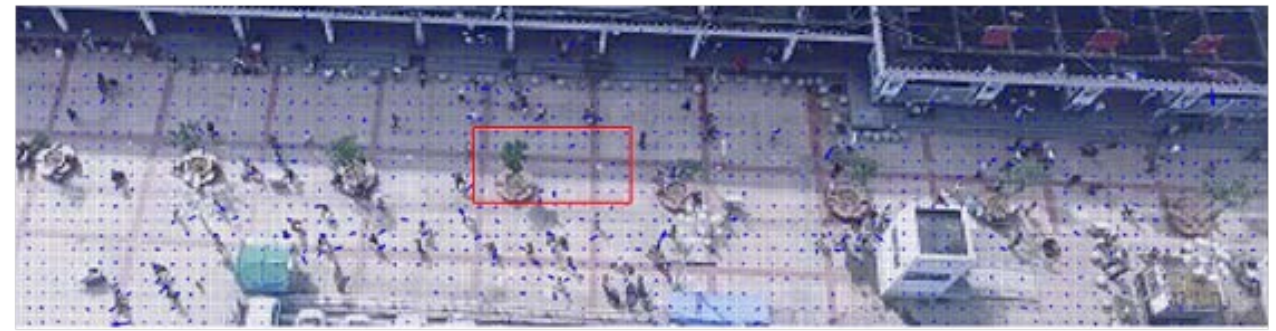

Fig.5 velocity field before Gaussian filter 


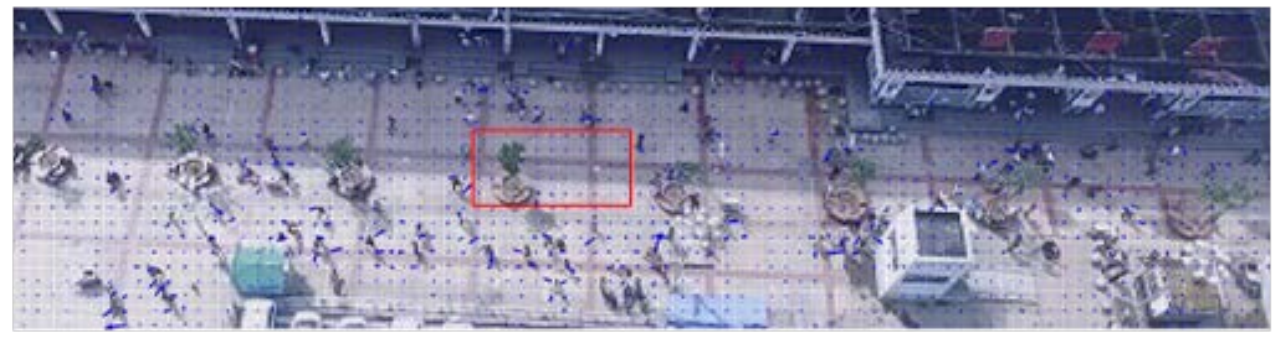

Fig.6 velocity field after Gaussian filter

Compared Fig.5 and Fig.6, we can find that the area velocity which has no pedestrians improves effectively. The velocity information is mainly focused on the pedestrian movement characteristics. In order to further instructions, we study the red square area detailedly. Fig.7 and Fig.8 are experimental results.

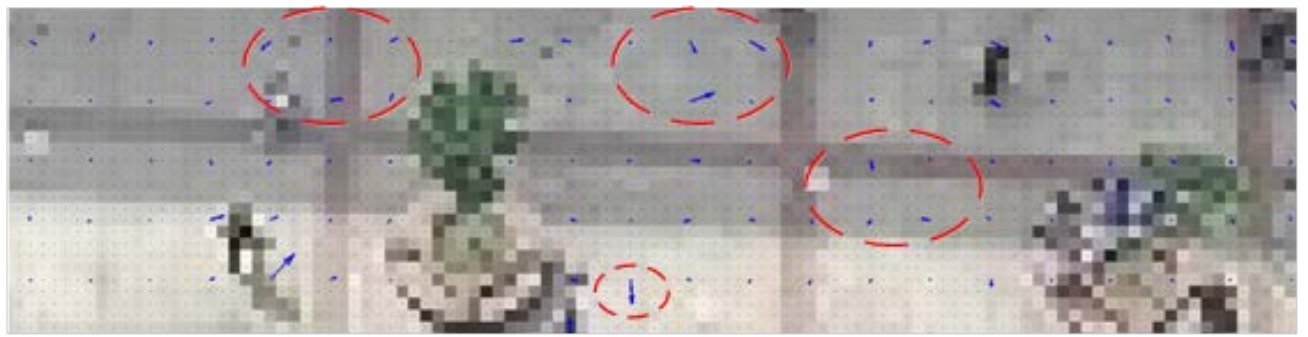

Fig.7 Local amplification figure before Gaussian filter

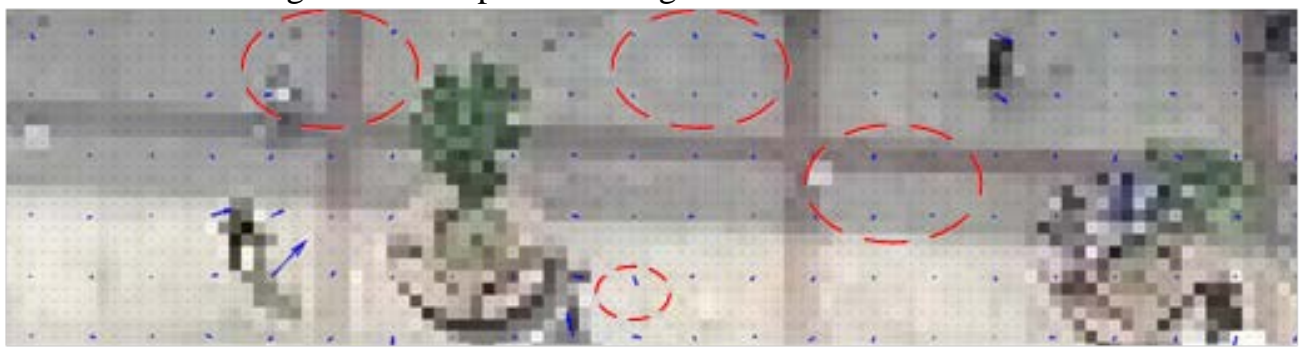

Fig.8 Local amplification figure after Gaussian filter

Paying attention to the velocity of dotted red circle in Fig.7 and Fig.8, we can see that the velocity generated by noise has been eliminated after Gaussian filter. It is helpful for us to construct better velocity field.

For crowd velocity vector analysis, we can observe the pedestrians velocity vector through amplification Fig.6. Fig.9 is experimental results.

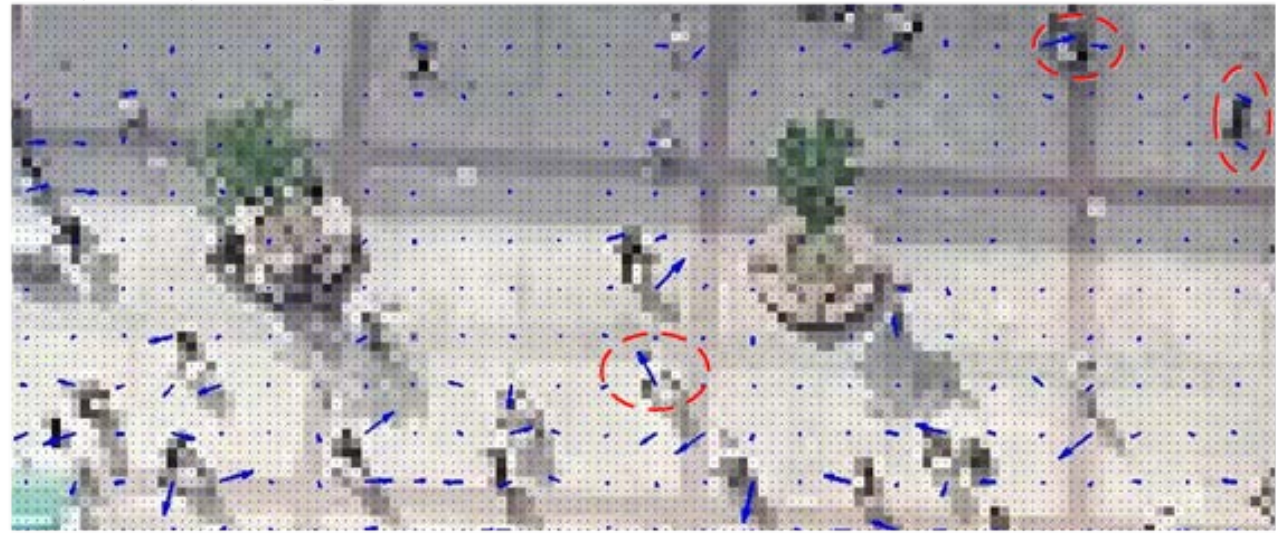

Fig.9 Local amplification figure after Gaussian filter

Fig.9 shown that it can ideally reflect pedestrians movement characteristics by Horn-Schunck algorithm and Gaussian filter. We orderly extract the pedestrian velocity vector of dotted red circle from left to right from the velocity vector matrix computed by MATLAB. They orderly are -1.0404, $0.9461,0.4714$.

Fig.10 is our constructed velocity field. 


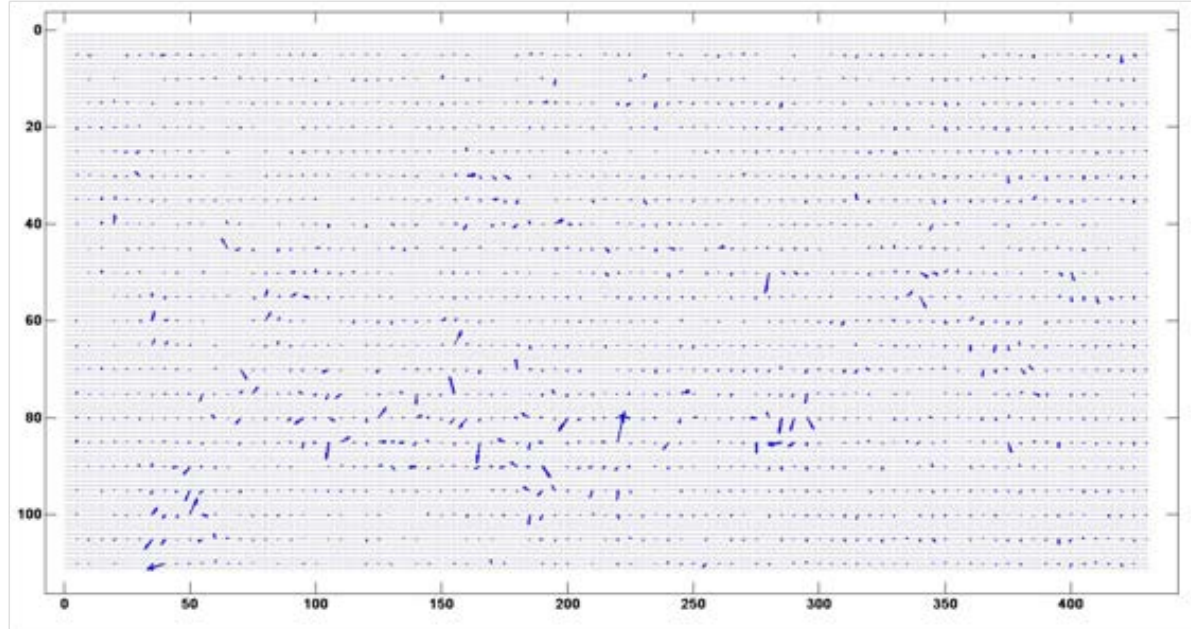

Fig.10 velocity field

velocity field design experimental. We set the threshold velocity $V_{\min }=0.4 \mathrm{~m} / \mathrm{s}$ to eliminate velocity of scene. And we can get velocity field as shown in Fig.11.

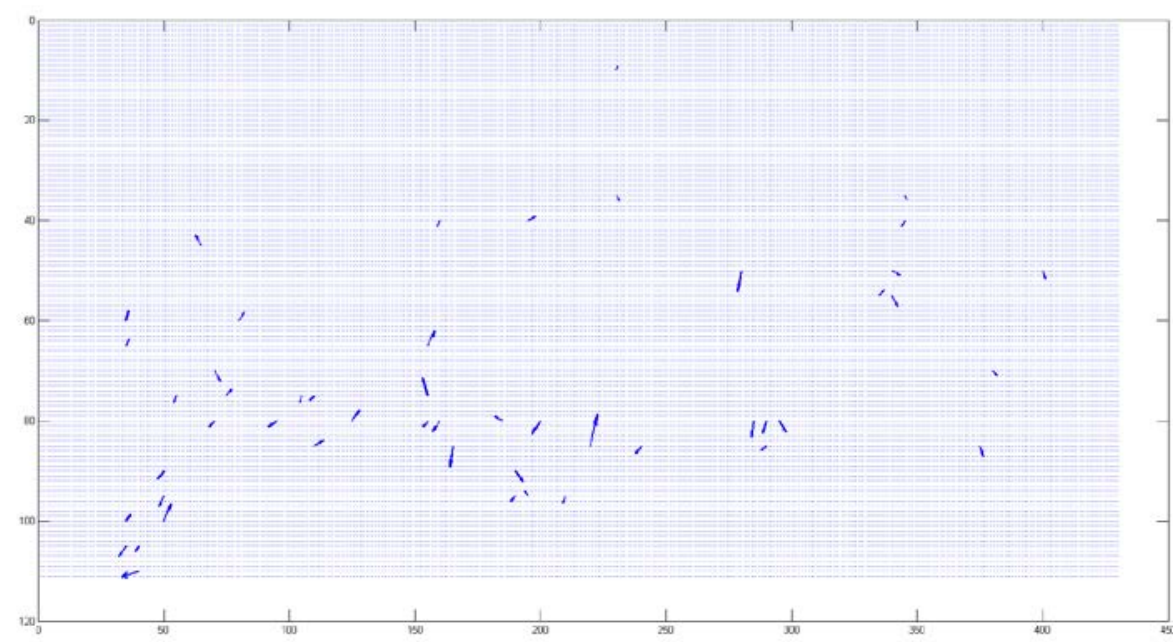

Fig.11 velocity field without scene

And then, we extract the sample velocity vector in Fig.11, and add some sample points artificially. Finally, we can construct the velocity field generated by two hundred sample points (black triangle arrow) as shown in Fig.12.

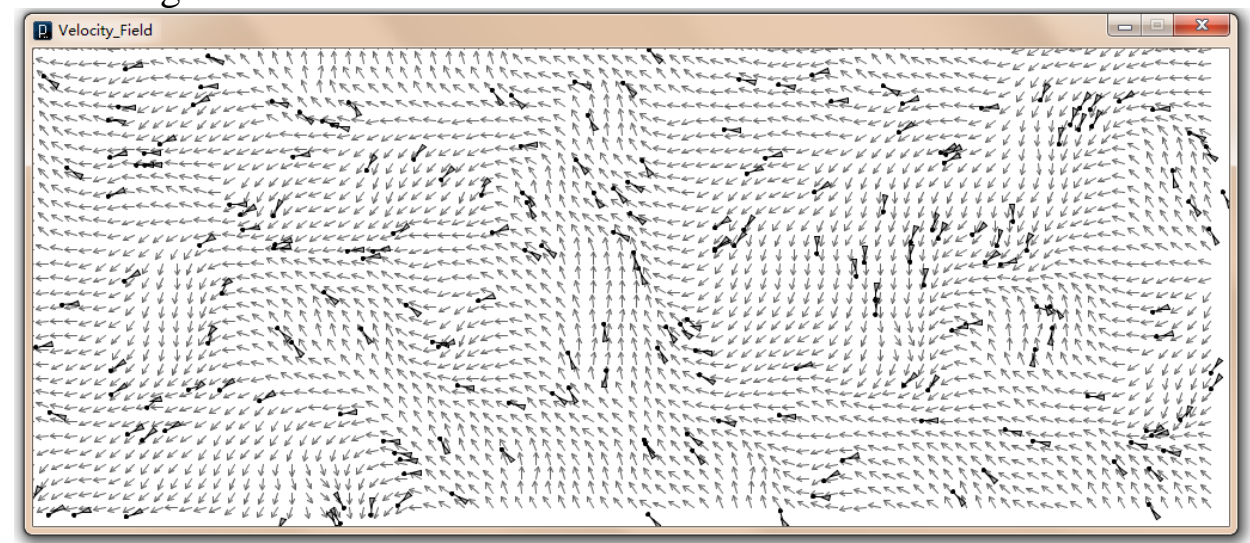

Fig.12 velocity field generated by sample points

\section{Summary}

In this paper, we present the optimization on the limitations that virtual crowd simulation scenario should be consistent with the crowd scene of video data. And then, we acquire and process the crowd 
video in the Changsha railway station square to generate velocity field. Next, we design velocity field by the presented optimization strategy. Finally, we construct the ideal velocity field.

Several interesting issues regarding the crowd behavior model require further exploration.

First, video data acquisition is still difficulty[8]. It often encounter all sorts of problems in the process of real data acquisition. Such as: Shooting angle and location, The shaking of the camera, etc. So we need to study more optimization algorithm to extract pedestrians behavior.

Second, the driven mehtod should be combinational each other[8]. In crowd behavior simulation, we can be classified as model-driven and data-driven modeling method according to the focuses and scale of crowd. While in present resarch, we need to study the crowd behavior form different focuses simultaneously. So the combination of the model-driven and data-driven methods is our major work in future research.

\section{Acknowledgment}

The research work was supported by National Natural Science Foundation of China under Grant No. 61170160.

\section{References}

[1] Musse, Soraia R., et al. "Using computer vision to simulate the motion of virtual agents." Computer Animation and Virtual Worlds 18.2 (2007): 83-93.

[2] Treuille, Adrien, Seth Cooper, and Zoran Popović. "Continuum crowds." ACM Transactions on Graphics (TOG) 25.3 (2006): 1160-1168.

[3] Courty, Nicolas, and Thomas Corpetti. "Crowd motion capture." Computer Animation and Virtual Worlds 18.4 - 5 (2007): 361-370.

[4] Andrade, Ernesto L., Scott Blunsden, and Robert B. Fisher. "Hidden markov models for optical flow analysis in crowds." Pattern Recognition, 2006. ICPR 2006. 18th International Conference on. Vol. 1. IEEE, 2006.

[5] Lerner, Alon, Yiorgos Chrysanthou, and Dani Lischinski. "Crowds by example." Computer Graphics Forum. Vol. 26. No. 3. Blackwell Publishing Ltd, 2007.

[6] Horn, Berthold K., and Brian G. Schunck. "Determining optical flow." 1981 Technical symposium east. International Society for Optics and Photonics, 1981.

[7] Jin, Xiaogang, et al. "Interactive control of large-crowd navigation in virtual environments using vector fields." IEEE Computer Graphics and Applications 6 (2008): 37-46.

[8] Thalmann, Daniel, et al. "Challenges in crowd simulation." 2009 International Conference on CyberWorlds. IEEE, 2009. 